\title{
ON A THEOREM OF MUCKENHOUPT AND WHEEDEN AND A WEIGHTED INEQUALITY RELATED TO SCHRÖDINGER OPERATORS
}

\author{
C. PÉREZ
}

\begin{abstract}
We extend in several directions a theorem of B. Muckenhoupt and $\mathrm{R}$. Wheeden relating the $L^{p}$-norms of Riesz potentials and fractional maximal operators. We apply these results to give a simple proof and sharpen a weighted inequality for Schrödinger operators of Chang, Wilson and Wolff.
\end{abstract}

\section{INTRODUCTION}

The main purpose of this paper is to give a simple proof and to sharpen a weighted inequality for Schrödinger operators obtained by A. Chang, M. Wilson and T. Wolff in [6]. Our approach is different, and it is based on several extensions of an important theorem of B. Muckenhoupt and R. Wheeden (cf. [19]) relating the Riesz potentials and the fractional maximal operators. The idea of using Muckenhoupt-Wheeden's theorem for studying these questions is taken from M. Schechter in [22].

To be precise we let $I_{\beta}, 0<\beta<n$, denote the Riesz potentials or fractional integrals defined by

$$
I_{\beta} f(x)=\int_{\mathbb{R}^{n}} \frac{f(y)}{|x-y|^{n-\beta}} d y .
$$

These operators are closely related to the Marcinkiewicz or fractional maximal operators $M_{\beta}$ defined by

$$
M_{\beta} f(x)=\sup _{x \in Q} \frac{1}{|Q|^{1-\beta / n}} \int_{Q}|f(y)| d y,
$$

where the supremum is taken over all cubes $Q$. The definition readily gives the pointwise inequality $M_{\beta} f(x) \leq c I_{\beta} f(x), x \in \mathbb{R}^{n}$. However, the operator $I_{\beta}$ is not much larger than $M_{\beta}$ as the theorem of B. Muckenhoupt and R. Wheeden mentioned above shows. We recall that a weight $w$ satisfies the $A_{\infty}$ condition of Muckenhoupt if there are constants $C$ and $\delta>0$ such that

$$
\frac{w(E)}{w(Q)} \leq c\left(\frac{|E|}{|Q|}\right)^{\delta},
$$

for each cube $Q$ and each measurable subset $E \subset Q$.

Received by the editors July 17,1990 and, in revised form, September 28, 1990.

1980 Mathematics Subject Classification (1985 Revision). Primary 42B25, 42B20, 42B15, 81C10. 
Theorem 1.1 (Muckenhoupt-Wheeden). Let $0<\beta<n, 0<p<\infty$, and let $w \in A_{\infty}$. Then

$$
\left\|I_{\beta} f\right\|_{L^{p}(w)} \approx\left\|M_{\beta} f\right\|_{L^{p}(w)}
$$

for each function $f \in C_{0}^{\infty}$.

In recent years this theorem has found several interesting applications. For instance in [1] D. Adams has provided a simpler proof of the celebrated $T$. Wolff's inequality in nonlinear potential theory than that in [12]. Another interesting realization of Muckenhoupt-Wheeden's theorem is that it is essentially equivalent with the fact that the positive cone of the Triebel-Lizorkin spaces $\dot{\mathrm{F}}_{p}^{\alpha, q}, \alpha<0,0<p, q \leq \infty$ is independent of the parameter $q$ (cf. [15]). As we mentioned above M. Schechter used it (cf. [22]) to give a simpler proof of an estimate for Schrödinger operators due to C. Fefferman and D. H. Phong (cf. below). ing:

The weighted inequality obtained by Chang, Wilson and Wolff is the follow-

Theorem 1.2. Let $\varphi:(0, \infty) \rightarrow(0, \infty)$ be an increasing function, such that for some positive constant $c$,

$$
\int_{c}^{\infty} \frac{1}{\varphi(t)} \frac{d t}{t}<\infty .
$$

Suppose that $v$ is a nonnegative function on $\mathbb{R}^{n}$ such that for every cube $Q$

$$
\frac{|Q|^{2 / n}}{|Q|} \int_{Q} v(y)^{2} \varphi\left(v(y)^{2}|Q|^{2 / n}\right) d y \leq k,
$$

with $k$ independent of $Q$. Then for each $f \in C_{0}^{\infty}$,

$$
\int_{\mathbb{R}^{n}}(v(y)|f(y)|)^{2} d y \leq C \int_{\mathbb{R}^{n}}|\nabla f(y)|^{2} d y,
$$

where the constant $C$ is a multiple of $k$, but independent of $f$.

Shortly afterwards S. Chanillo and R. Wheeden apply the method of [6] to extend this result to the $L^{p}$ case. This has been done in [7]. They also consider a weighted version of (3) by replacing the Lebesgue measure $d x$ on the righthand side by the weighted measure $w(x) d x$, with the assumption that $w \in A_{p}$. J. M. Wilson has obtained in [25] Chanillo-Wheeden's theorem by deriving first a two weighted square function inequality.

If we let $\varphi(t)=t^{r-1}, 1<r<\infty$, the condition on $v$ becomes

$$
|Q|^{1 / n}\left(\frac{1}{|Q|} \int_{Q} v(y)^{2 r} d y\right)^{1 / 2 r} \leq k
$$

These classes of weights were introduced by C. Fefferman and D. H. Phong in [9] in connection with the spectrum of the Schrödinger operator $-\Delta-v$, and in particular they obtained (3) for $\varphi(t)=t^{r-1}$. The weights studied by C. Fefferman and D. H. Phong are particular cases of the Morrey spaces which play a central role in the regularity theory of nonlinear elliptic equations (cf. [13]), and more recently in the initial value problem for the Navier-Stokes equations (cf. [14]). The approach used by Fefferman and Phong is based on (difficult) estimates of certain square functions from Harmonic Analysis. Shortly afterward 
Chang, Wilson and Wolff took up the same approach to obtain Theorem 1.2. On the other hand R. Kerman and E. Sawyer obtained in [16], and by different means, the following less geometrical but necessary and sufficient condition on $v$ for inequality (3) to hold:

$$
\int_{Q} \int_{Q} \frac{v(x)^{2} v(y)^{2}}{|x-y|^{n-2}} d x d y \leq k v^{2}(Q),
$$

uniformly on each dyadic cube $Q$, where $v^{2}(Q)=\int_{Q} v(y)^{2} d y$.

The paper is organized as follows. In $\S 2$ we state the main results of the paper. In $\S 3$ we collect some known results and set some notations that will be used in the sequel. In $\S 4$ we shall prove several extensions of the MuckenhouptWheeden theorem, and finally in $\S 5$ we apply these results to obtain a weighted inequality for Schrödinger operators.

Acknowledgment. The author wants to thank Professor R. Kerman for proposing he look for a different proof of Theorem 1.2. He also is grateful to Professor E. Sawyer for his interest in this work, and to the referee for pointing out to him several interesting references.

\section{Statement of THE Results}

Our first result shows that Theorem 1.1 for the Lebesgue measure $w=1$ still holds if we replace the $L^{p}$ space norm by more general Banach function space norms. For an arbitrary Banach function space $X, X^{\prime}$ denotes it dual, which is also another Banach function space.

Theorem 2.1. Let $X$ be any rearrangement-invariant Banach function space on $\mathbb{R}^{n}$ with respect to the Lebesgue measure. Suppose further that the Boyd upper index $\bar{\alpha}_{X^{\prime}}$ is so that $\bar{\alpha}_{X^{\prime}}<1$. Then

$$
\left\|I_{\beta} f\right\|_{X} \approx\left\|M_{\beta} f\right\|_{X}, \quad f \in C_{0}^{\infty} .
$$

We shall also extend Theorem 1.1 to the vector-valued setting.

Theorem 2.2. Let $1<p, q<\infty$. Then

$$
\left\|\left(\sum_{i=0}^{\infty}\left|I_{\beta} f_{i}\right|^{q}\right)^{1 / q}\right\|_{L^{p}} \approx\left\|\left(\sum_{i=0}^{\infty}\left|M_{\beta} f_{i}\right|^{q}\right)^{1 / q}\right\|_{L^{p}},
$$

for all functions $f_{i} \in C_{0}^{\infty}$.

We remark that none of the above results follow directly from the good- $\lambda$ type estimate obtained in [19].

In order to state our extension of Theorem 1.2 we shall define the following general average. Let $X$ be a Banach function space over $\mathbb{R}^{n}$ with respect to the Lebesgue measure. Given a measurable function $f$ and any cube $Q$ we define the $X$-average of $f$ over $Q$ by

$$
\|f\|_{X, d Q}=\left\|\tau_{l(Q)}\left(f \chi_{Q}\right)\right\|_{X}
$$

where $\tau_{\delta}, \delta>0$, is the dilation operator $\tau_{\delta} f(x)=f(\delta x)$, and $\chi_{E}$ is a characteristic function of $E$. Observe that in the case $X=L^{r}$ the average is

$$
\left(\frac{1}{|Q|} \int_{Q} w(y)^{r} d y\right)^{1 / r} \text {. }
$$


More generally, let $X=L^{B}$ be the Orlicz space defined by the Young function $B$. Then the $B$-average is given by

$$
\|f\|_{B, d Q}=\inf \left\{\lambda>0: \frac{1}{|Q|} \int_{Q} B\left(\frac{|f(y)|}{\lambda}\right) d y \leq 1\right\} .
$$

For any Banach function space $X$ we associate the following maximal operator defined for each locally integrable function $f$ by

$$
M_{X} f(x)=\sup _{x \in Q}\|f\|_{X, d Q},
$$

where the supremum is taken over all the cubes containing $x$.

Theorem 2.3. Let $1<p<\infty$, and let $X$ be a Banach function space such that $M_{X^{\prime}}: L^{p^{\prime}}\left(\mathbb{R}^{n}\right) \rightarrow L^{p^{\prime}}\left(\mathbb{R}^{n}\right)$. Suppose that $v$ is a nonnegative function on $\mathbb{R}^{n}$ such that for every cube $Q$,

$$
|Q|^{1 / n}\|v\|_{X, d Q} \leq k
$$

with $k$ independent of $Q$. Then for each $f \in C_{0}^{\infty}$,

$$
\int_{\mathbb{R}^{n}}(v(y)|f(y)|)^{p} d y \leq C \int_{\mathbb{R}^{n}}|\nabla f(y)|^{p} d y,
$$

where the constant $C$ is a multiple of $k$, but independent of $f$.

To give a result in terms of Orlicz norms we give the following definition.

Definition 2.4. Let $1<p<\infty$. We say that a Young function $B$ belongs to the class $B_{p}$ or that it satisfies the $B_{p}$ condition if there is a positive constant $c$ for which

$$
\int_{c}^{\infty} \frac{B(t)}{t^{p}} \frac{d t}{t}<\infty
$$

We observe that if $1<p<q<\infty$, then $B_{p} \subset B_{q}$.

Remark 2.5. Sometimes it is more convenient to deal with the complementary function $\bar{B}$ of $B$. Assuming that $B$ is doubling, it can be checked using (18) and (19) that the Young function $B$ satisfies the $B_{p}$ condition if and only if there is a positive constant $c$ for which

$$
\int_{c}^{\infty}\left(\frac{t^{p^{\prime}}}{\bar{B}(t)}\right)^{p-1} \frac{d t}{t}<\infty .
$$

We also point out that we get an equivalent definition if we replace $c$ by 1 .

Example 2.6. Let $1<p<\infty$. A typical Young function that belongs to the class $B_{p}$ is $B(t)=t^{s}$ with $1 \leq s<p$. Another more interesting example is when considering $B$ such that

$$
\bar{B}(t) \approx t^{p^{\prime}} \log ^{p^{\prime}-1+\delta}(1+t),
$$

or

$$
\bar{B}(t) \approx t^{p^{\prime}} \log ^{p^{\prime}-1}(1+t)[\log \log (1+t)]^{p^{\prime}-1+\delta},
$$

with $\delta>0$. Then $B \in B_{p}$ by the above remark.

Our $L^{p}$ version of Theorem 1.2 is 
Corollary 2.7. Let $1<p<\infty$, and let $B$ be a Young function such that $\bar{B} \in B_{p^{\prime}}$. Suppose that $v$ is a nonnegative function on $\mathbb{R}^{n}$ such that for every cube $Q$

$$
|Q|^{1 / n}\|v\|_{B, d Q} \leq k
$$

with $k$ independent of $Q$. Then for each $f \in C_{0}^{\infty}$,

$$
\int_{\mathbb{R}^{n}}(v(y)|f(y)|)^{p} d y \leq C \int_{\mathbb{R}^{n}}|\nabla f(y)|^{p} d y,
$$

where the constant $C$ is a multiple of $k$, but independent of $f$.

We point out that this result is contained in Theorem (1.3) of [7] and in Theorem 4.1 of [25].

Let us show that this result contains Theorem 1.2. Let $p=2$ and $k$, and $\varphi$ be as in Theorem 1.2. Let us consider the Young function $B(t)=$ $k \int_{0}^{t} s \varphi\left(k^{2} s^{2}\right) d s$. Since $\varphi$ is increasing, the condition (2) implies

$$
\frac{1}{|Q|} \int_{Q} B\left(\frac{v(y)|Q|^{1 / n}}{k}\right) d y \leq 1 .
$$

However, by the definition of the Luxemburg norm (7) this is equivalent with

$$
\left\|\frac{v|Q|^{1 / n}}{k}\right\|_{B, d Q} \leq 1, \quad \text { or }|Q|^{1 / n}\|v\|_{B, d Q} \leq k,
$$

and this is condition (11).

A corresponding result for Lorentz spaces is the following.

Corollary 2.8. Let $1<r, p<\infty$. Suppose that $v$ is a nonnegative function on $\mathbb{R}^{n}$ such that for every cube $Q$,

$$
|Q|^{1 / n} \frac{1}{|Q|^{1 / r p}}\left\|\chi_{Q} v\right\|_{L^{r p, \infty}} \leq k,
$$

with $k$ independent of $Q$. Then for each $f \in C_{0}^{\infty}$,

$$
\int_{\mathbb{R}^{n}}(v(y)|f(y)|)^{p} d y \leq C \int_{\mathbb{R}^{n}}|\nabla f(y)|^{p} d y,
$$

where the constant $C$ is a multiple of $k$, but independent of $f$.

We can give a vector-valued version of Theorem 2.3 as follows.

Theorem 2.9. Let $1<p, q<\infty$, and let $B$ be a Young function such that $\bar{B} \in B_{\min \left\{p^{\prime}, q^{\prime}\right\}}$. Suppose that $v$ is a nonnegative function on $\mathbb{R}^{n}$ such that for every cube $Q$,

$$
|Q|^{1 / n}\|v\|_{B, d Q} \leq k,
$$

with $k$ independent of $Q$. Then for each $f_{i} \in C_{0}^{\infty}$,

$$
\left\|\left(\sum_{i=0}^{\infty}\left|f_{i}\right|^{q}\right)^{1 / q} v\right\|_{L^{p}} \leq c\left\|\left(\sum_{i=0}^{\infty}\left|\nabla f_{i}\right|^{q}\right)^{1 / q}\right\|_{L^{p}},
$$

where the constant $C$ is a multiple of $k$, but independent of $f_{i}$.

For the proof of this theorem we shall make use of the following lemma. 
Lemma 2.10. Let $1<p, q<\infty$, and let $B$ be a Young function such that $B \in B_{\min \{p, q\}}$. Then there is a constant $c=c_{p, q}$ such that for each $f_{i} \in C_{0}^{\infty}$,

$$
\left\|\left(\sum_{i=0}^{\infty}\left(M_{B} f_{i}\right)^{q}\right)^{1 / q}\right\|_{L^{p}} \leq c\left\|\left(\sum_{i=0}^{\infty}\left|f_{i}\right|^{q}\right)^{1 / q}\right\|_{L^{p}} .
$$

Of course this lemma contains the classical Fefferman-Stein vector-valued inequality when considering $B(t)=t$ (cf. [10]).

\section{Preliminaries}

In this section we shall provide some background from the theory of function spaces that will be used later. We begin by recalling some basic facts about the theory of Banach function spaces. We shall refer to [3] for a more complete account. Let $(R, \mu)$ be a measure space, and let $M^{+}(R)$ be the cone of $\mu$-measurable functions on $R$ whose values lie in $[0, \infty]$. A mapping $\rho: M^{+}(R) \rightarrow[0, \infty]$ is called a Banach function norm if, for all $f, g, f_{n}$ $(n=1,2,3, \ldots)$ in $M^{+}(R)$, for all constants $a \geq 0$, and for all $\mu$-measurable subsets $E$ of $R$, the following properties hold:

(i) $\quad \rho(f)=0$ iff $f=0 \quad \mu$-a.e.; $\rho(a f)=a \rho(f)$; $\rho(f+g) \leq \rho(f)+\rho(g)$,

(ii) $0 \leq g \leq f$, $\mu$-a.e. implies $\rho(g) \leq \rho(f)$,

(iii) $0 \leq f_{n} \uparrow f$, $\mu$-a.e. implies $\rho\left(f_{n}\right) \uparrow \rho(f)$,

(iv) $\mu(E)<\infty$ implies $\rho\left(\chi_{E}\right)<\infty$,

(v) $\mu(E)<\infty$ implies $\int_{E} f d \mu \leq C_{E} \rho(f)$,

for some constant $C_{E}, 0<C_{E}<\infty$, depending on $E$ and $\rho$ but independent of $f$. Let $M(R)$ denote the collection of all $\mu$-measurable functions on $R$. The collection $X=X(\rho)$ of all functions $f \in M(R)$ for which $\rho(|f|)=\|f\|_{X}<\infty$ is called a Banach function space. The most important property of the Banach function spaces that we shall be using is as follows. Given a Banach function space $X$ there is another Banach function space $X^{\prime}$, the associate space of $X$, for which the following generalized Hölder inequality holds:

$$
\int_{R}|f(y) g(y)| d \mu(y) \leq\|f\|_{X}\|g\|_{X^{\prime}} .
$$

A Banach function space $X$ is said to be rearrangement-invariant if whenever $f, g \in X$ are equimeasurable, then $\|f\|_{x}=\|g\|_{X}$. Recall that two functions are equimeasurable if $\mu_{f}(t)=\mu_{g}(t), t>0$, where

$$
\mu_{f}(t)=\mu\{x \in R:|f(x)|>t\},
$$

is the distribution of $f$. Most of the properties of the rearrangement-invariant spaces can be formulated in terms of the fundamental function of $X, \varphi$, given by

$$
\varphi_{X}(t)=\left\|\chi_{E}\right\|_{X},
$$

where $\mu(E)=t$. Observe that the particular choice of the set $E$ with $\mu(E)=t$ is immaterial by the rearrangement-invariance of $X . \varphi_{X}$ is quasiconcave and continuous, except perhaps at the origin. Furthermore, if $X^{\prime}$ is the associate space of $X$ the following identity holds:

$$
\varphi_{X}(t) \varphi_{X^{\prime}}(t)=t, \quad t>0 .
$$


Examples of rearrangement-invariant spaces include the Lebesgue $L^{p}$ spaces, the Lorentz $\Lambda, M$, and $L^{p, q}$ spaces (cf. [3]), and the Orlicz spaces that we are going to describe briefly next.

The Orlicz spaces are one of the most relevant rearrangement-invariant Banach function spaces. We shall provide some basic facts about these spaces, and refer to the classical reference [17] or to [18, Chapter 3] for a general account. A function $B$ defined on $[0, \infty)$ is a Young function if it is continuous, convex and increasing and satisfying $B(0)=0$ and $B(t) \rightarrow \infty$ as $t \rightarrow \infty$. We shall assume that $B$ is normalized so that $B(1)=1$. We shall require that $B$ satisfies the $\Delta_{2}$ condition, namely that there are constants $C>0, k \geq 0$ so that

$$
B(2 t) \leq C B(t), \quad t \geq k \geq 0 .
$$

Each Young function $B$ has associated a complementary Young function $\bar{B}$ that satisfies

$$
t \leq B^{-1}(t) \bar{B}^{-1}(t) \leq 2 t, \quad t>0 .
$$

Also we shall make use of the following property without explicit reference:

$$
B(t) \approx t \frac{d B}{d t}(t), \quad t>0 .
$$

Let $(X, \mu)$ be a measure space and let $B$ be a Young function. The Orlicz space $L^{B}(\mu)$ consists of all $\mu$-measurable functions $f$ such that

$$
\int_{X} B\left(\frac{|f(y)|}{\lambda}\right) d \mu(y)<\infty,
$$

for some $\lambda>0 . L^{B}(\mu)$ can be normed by the Luxemburg norm defined by

$$
\|f\|_{B, \mu}=\inf \left\{\lambda>0: \int_{X} B\left(\frac{|f(y)|}{\lambda}\right) d \mu(y) \leq 1\right\} .
$$

$L^{B}(\mu)$ equipped with the Luxemburg norm is a rearrangement-invariant space with fundamental function given by $\varphi_{B}(t)=\varphi_{L^{B}(\mu)}(t)=B^{-1}\left(\frac{1}{t}\right)^{-1}$.

Next we shall state the theorem which characterizes those rearrangementinvariant spaces for which the Hardy-Littlewood maximal operator $M$ is bounded. These spaces are characterized in terms of the Boyd upper index $\bar{\alpha}_{X}$, and we refer the reader to [3, p. 149] for the definition. We just mention that the Lebesgue $L^{p}$ spaces, or more generally, the Lorentz spaces $L^{p, q}$ have Boyd index equal to $\frac{1}{p}$. Also for a Young function $B$, the Boyd upper index of the Orlicz space $L^{B}(\mu)$ is given by

$$
\bar{\alpha}_{L^{B}(\mu)}=\lim _{t \rightarrow \infty} \frac{\log g(t)}{\log t}
$$

where $g$ is defined by

$$
g(t)=\limsup _{s \rightarrow \infty} \frac{B^{-1}(s)}{B^{-1}\left(\frac{s}{t}\right)} .
$$

Theorem 3.1 (G. G. Lorentz-T. Shimogaki). Let $X$ be a rearrangement-invariant space on $\mathbb{R}^{n}$. Then the Hardy-Littlewood maximal operator $M$ is bounded on $X$ if and only if the upper index of $X$ satisfies $\bar{\alpha}_{X}<1$.

For the proof we refer the reader to [3, Chapter 3]. 
Example 3.2. Let $1<p<\infty$, then the Lebesgue $L^{p}$ spaces, or Lorentz $L^{p, q}$ spaces, have Boyd index less than 1. Also, the same is true for any Orlicz space $L^{B}$ with Young function defined by $B(t) \approx t^{p} \log ^{m}(1+t), p>1, m \in \mathbb{R}$.

We shall conclude the section recalling some basic definitions from the theory of weighted norm inequalities that will be needed later. We say that the weight $w$ belongs to the Muckenhoupt class $A_{p}, 1<p<\infty$, if there is a constant $c$ such that

$$
\left(\frac{1}{|Q|} \int_{Q} w(y) d y\right)^{1 / p}\left(\frac{1}{|Q|} \int_{Q}(w) y^{1-p^{\prime}} d y\right)^{1 / p^{\prime}} \leq c
$$

uniformly for all cubes $Q$. For $1<p<\infty, p^{\prime}$ will always denote the dual of $p$, that is $\frac{1}{p}+\frac{1}{p^{\prime}}=1$. In the limit case $p=1$, we have

$$
\left(\frac{1}{|Q|} \int_{Q} w(y) d y\right) \underset{Q}{\text { ess. } \sup \left(w^{-1}\right) \leq c}
$$

for all $Q$; this is equivalent to saying $M w(x) \leq c w(x)$ for almost every $x \in$ $\mathbb{R}^{n}$. For the other limit case, $p=\infty$, we have that

$$
A_{\infty}=\bigcup_{p>1} A_{p}
$$

which is equivalent with the definition (1) given in the first section. For a general introduction to the subject, and for historical comments as well we refer the reader to [11].

\section{PROOFS OF THE EXTENSIONS OF THE MUCKENHOUPT-WHEEDEN THEOREM}

Proof of Theorem 2.1. Since $I_{\beta}$ is a positive operator we may assume that $f$ is nonnegative. By duality there exists a nonnegative function $g \in X^{\prime}$ with unit norm and such that

$$
\left\|I_{\beta} f\right\|_{X}=\int_{\mathbb{R}^{n}} I_{\beta} f(y) g(y) d y .
$$

We adapt now the so-called Rubio de Francia algorithm (cf. [11, Lemma 4.1 in Chapter 4]). The assumption $\bar{\alpha}_{X^{\prime}}<1$ yields the boundedness of the HardyLittlewood maximal function $M$ as an operator on $X^{\prime}$ by Theorem 3.1. Let $0<A<\infty$ be the norm of that operation. Define

$$
w=\sum_{i=0}^{\infty} \frac{M^{i} g}{(2 A)^{i}}
$$

where $M^{0}=I_{d}$ and $M^{i}$ is the $i$ th iterate of the operator $M$. Hence, $g(x) \leq$ $w(x),\|w\|_{X^{\prime}} \leq 2\|g\|_{X^{\prime}}=2$, and furthermore $w \in A_{1}$. Therefore, by Theorem 1.1 and the generalized Hölder inequality (15)

$$
\begin{aligned}
\left\|I_{\beta} f\right\|_{X} & \leq \int_{\mathbb{R}^{n}} I_{\beta} f(y) w(y) d y \leq C \int_{\mathbb{R}^{n}} M_{\beta} f(y) w(y) d y \\
& \leq C\left\|M_{\beta} f\right\|_{X}\|w\|_{X^{\prime}} \leq C\left\|M_{\beta} f\right\|_{X} .
\end{aligned}
$$

This concludes the proof of the theorem. 
Proof of Theorem 2.2. The vector-valued (5) is obvious for $q=p$ by Theorem 1.1 with $w=1$. We shall use some well-known duality arguments. Suppose first that $1<q<p<\infty$. Then we can write

$$
\left\|\left(\sum_{i=0}^{\infty}\left(I_{\beta} f_{i}\right)^{q}\right)^{1 / q}\right\|_{L^{p}}^{q}=\left\|\sum_{i=0}^{\infty}\left(I_{\beta} f_{i}\right)^{q}\right\|_{L^{p / q}}=\sum_{i=0}^{\infty} \int_{\mathbb{R}^{n}} I_{\beta} f_{i}(x)^{q} g(x) d x,
$$

for some $0 \leq g \in L^{t}$ with unit norm, where $t=\left(\frac{p}{q}\right)^{\prime}>1$. Let $1<r<t$. Then since $M\left(g^{r}\right)^{1 / r} \in A_{1}$ we have by Theorem 1.1 that

$$
\begin{aligned}
& \left\|\left(\sum_{i=0}^{\infty}\left(I_{\beta} f_{i}\right)^{q}\right)^{1 / q}\right\|_{L^{p}}^{q} \leq C \sum_{i=0}^{\infty} \int_{\mathbb{R}^{n}} M_{\beta} f_{i}(x)^{q} M\left(g^{r}\right)(x)^{1 / r} d x \\
& \quad \leq C\left\|\sum_{i=0}^{\infty}\left(M_{\beta} f_{i}\right)^{q}\right\|_{L^{t^{\prime}}}\left\|M\left(g^{r}\right)^{1 / r}\right\|_{L^{t}}=C\left\|\left(\sum_{i=0}^{\infty}\left(M_{\beta} f_{i}\right)^{q}\right)^{1 / q}\right\|_{L^{p}}^{q}\left\|M\left(g^{r}\right)\right\|_{L^{t / r}}^{1 / r} .
\end{aligned}
$$

Since $t>r$, the Hardy-Littlewood maximal theorem, and the fact that $\|g\|_{L^{t}}=$ 1 give the desired result.

Let us suppose now that $1<p<q<\infty$, then we can write

$$
\left\|\left(\sum_{i=0}^{\infty}\left(M_{\beta} f_{i}\right)^{q}\right)^{1 / q}\right\|_{L^{p}}^{q}=\left\|\sum_{i=0}^{\infty}\left(M_{\beta} f_{i}\right)^{q}\right\|_{L^{p / q}}=\sum_{i=0}^{\infty} \int_{\mathbb{R}^{n}} M_{\beta} f_{i}(x)^{q} \frac{1}{g(x)} d x,
$$

for some $0 \leq g \in L^{t}$ with unit norm, where $t=-\left(\frac{p}{q}\right)^{\prime}>0$ (cf. [11, p. 449]). Let $0<r<t$. Observe that this time it is not sure that $M\left(g^{r}\right)^{1 / r} \in A_{1}$, which would imply that $M\left(g^{r}\right)^{-1 / r} \in A_{2} \subset A_{\infty}$. However we have the following observation:

Lemma 4.1. Let $-\infty<\lambda<0$. Then $M(g)^{\lambda} \in A_{\infty}$.

For the proof simply take $s>1-\lambda$, and write $M(g)^{\lambda}=(M g)^{\frac{\lambda}{1-s}(1-s)}$ which belongs to $A_{s}$ since $\lambda /(1-s)<1$, and $s>1$ (cf. [11, p. 436]).

By this observation we can continue the proof of the theorem since $M\left(g^{r}\right)^{-1 / r}$ $\in A_{\infty}$. Hence, we can follow our estimate with

$$
\begin{aligned}
& \left\|\left(\sum_{i=0}^{\infty}\left(M_{\beta} f_{i}\right)^{q}\right)^{1 / q}\right\|_{L^{p}}^{q} \geq \sum_{i=0}^{\infty} \int_{\mathbb{R}^{n}} M_{\beta} f_{i}(x)^{q} \frac{1}{M\left(g^{r}\right)(x)^{1 / r}} d x \\
& \quad \approx \sum_{i=0}^{\infty} \int_{\mathbb{R}^{n}} I_{\beta} f_{i}(x)^{q} \frac{1}{M\left(g^{r}\right)(x)^{1 / r}} d x \geq\left\|\sum_{i=0}^{\infty}\left(I_{\beta} f_{i}\right)^{q}\right\|_{L^{p / q}}\left\|\frac{1}{M\left(g^{r}\right)^{1 / r}}\right\|_{L^{(\rho / q)^{\prime}}} \\
& \quad=\left\|\left(\sum_{i=0}^{\infty}\left(I_{\beta} f_{i}\right)^{q}\right)^{1 / q}\right\|_{L^{p}}^{q}\left\|M\left(g^{r}\right)^{1 / r}\right\|_{L^{t}}^{-1} .
\end{aligned}
$$

Here we have used Hölder's inequality with exponent $\alpha$ less than one, namely $\|f g\|_{L^{\prime}(\mu)} \geq\|f\|_{L^{\alpha}(\mu)}\|g\|_{L^{\alpha^{\prime}}(\mu)}$. Finally, since $\frac{t}{r}>1$ we apply again the HardyLittlewood maximal theorem, and the fact that $\|g\|_{L^{t}}=1$ to conclude the proof of the theorem. 


\section{THE WEIGHTED INEQUALITY FOR SCHRÖDINGER OPERATORS}

We shall start this section by recalling that for a Banach function space $X$ over $\mathbb{R}^{n}$ with respect to the Lebesgue measure, the $X$-average of any measurable function $f$ over any cube $Q$ is defined by

$$
\|f\|_{X, d Q}=\left\|\tau_{l(Q)}\left(f \chi_{Q}\right)\right\|_{X},
$$

where $\tau_{\delta}, \delta>0$, is the dilation operator $\tau_{\delta} f(x)=f(\delta x)$. Note that Hölder's inequality for Banach function spaces (15) yields after the change of variable $y=l(Q) z$

$$
\frac{1}{|Q|} \int_{Q} f(y) g(y) d y \leq\|f\|_{X, d Q}\|g\|_{X^{\prime}, d Q} .
$$

We also recall that the maximal operator $M_{X}$ is defined by

$$
M_{X} f(x)=\sup _{x \in Q}\|f\|_{X, d Q},
$$

where the supremum is taken over all the cubes containing $x$.

We shall use the following theorem that can be found in [20].

Theorem 5.1. Let $1<p<\infty$. Suppose that $B$ is a Young function. Then the following are equivalent.

$$
B \in B_{p}
$$

(ii) there is a constant $c$ such that

$$
\int_{\mathbb{R}^{n}} M_{B} f(y)^{p} d y \leq c \int_{\mathbb{R}^{n}} f(y)^{p} d y
$$

for all nonnegative, locally integrable functions $f$;

(iii) there is a constant $c$ such that

$$
\int_{\mathbb{R}^{n}} M_{B} f(y)^{p} w(y) d y \leq c \int_{\mathbb{R}^{n}} f(y)^{p} M w(y) d y
$$

for all nonnegative, locally integrable functions $f$ and $w$;

(iv) there is a constant $c$ such that

$$
\int_{\mathbb{R}^{n}} M f(y)^{p} \frac{w(y)}{\left[M_{\bar{B}}\left(u^{1 / p}\right)(y)\right]^{p}} d y \leq c \int_{\mathbb{R}^{n}} f(y)^{p} \frac{M w(y)}{u(y)} d y,
$$

for all nonnegative, locally integrable functions $f, w$ and $u$.

Proof of Theorem 2.3. We first claim that inequality (9) is equivalent to

$$
\int_{\mathbb{R}^{n}}\left(v(y) I_{1} f(y)\right)^{p} d y \leq C \int_{\mathbb{R}^{n}} f(y)^{p} d y
$$

for all nonnegative functions $f \in L^{p}\left(\mathbb{R}^{n}\right)$. That (28) is sufficient follows from the well-known pointwise inequality (cf. $[23$, p. 125]),

$$
f(x) \leq c I_{1}(|\nabla f|)(x), \quad x \in \mathbb{R}^{n},
$$

which holds for each $f \in C_{0}^{\infty}$. To prove the necessity we take any nonnegative functions $f \in L^{p}\left(\mathbb{R}^{n}\right)$, and test (9) with $g=I_{1} f$. However, the $L^{p}$-norm of the operator

$$
S f=\nabla I_{1} f
$$


is dominated by a multiple of sums of $L^{p}$-norms of Riesz transforms and thus $S$ is bounded on $L^{p}\left(\mathbb{R}^{n}\right)$ (cf. [23] or [11]). Hence this implies that (28) is necessary, and the claim is complete.

We shall prove now inequality (28). Since the adjoint operator of $f \rightarrow v I_{1}(f)$ is $f \rightarrow I_{1}(v f)$, by duality (28) is equivalent to

$$
\int_{\mathbb{R}^{n}} I_{1}(v f)(x)^{p^{\prime}} d x \leq C \int_{\mathbb{R}^{n}} f(x)^{p^{\prime}} d x, \quad 0 \leq f \in C_{0}^{\infty} .
$$

Now, by Theorem 1.1 it is also equivalent to

$$
\int_{\mathbb{R}^{n}} M_{1}(v f)(x)^{p^{\prime}} d x \leq C \int_{\mathbb{R}^{n}} f(x)^{p^{\prime}} d x .
$$

Now, to estimate the left-hand side of (30) we observe that (23) and (8) yield

$$
\frac{|Q|^{1 / n}}{|Q|} \int_{Q} v(y) f(y) d y \leq|Q|^{1 / n}\|v\|_{X, d Q}\|f\|_{X^{\prime}, d Q} \leq k\|f\|_{X^{\prime}, d Q},
$$

from which $M_{1}(v f)(x) \leq k M_{X^{\prime}} f(x), x \in \mathbb{R}^{n}$. Hence, the left-hand side of (30) is bounded by

$$
C \int_{\mathbb{R}^{n}} M_{X^{\prime}} f(x)^{p^{\prime}} d x .
$$

Finally, the assumption $M_{X^{\prime}}: L^{p^{\prime}}\left(\mathbb{R}^{n}\right) \rightarrow L^{p^{\prime}}\left(\mathbb{R}^{n}\right)$ concludes the proof of the theorem.

Proof of Corollary 2.7. Since it is assumed that $\bar{B} \in B_{p^{\prime}}$ we have from Theorem 5.1, equivalences (i) and (ii), that $M_{\bar{B}}: L^{p^{\prime}}\left(\mathbb{R}^{n}\right) \rightarrow L^{p^{\prime}}\left(\mathbb{R}^{n}\right)$. Finally, an application of Theorem 2.3 finishes the proof of the corollary.

Proof of Corollary 2.8. Let $X=L^{r p, \infty}$ with associated space $X^{\prime}=L^{(r p)^{\prime}, 1}$. The corollary follows from Theorem 2.3 if we show that $M_{(r p)^{\prime}, 1}: L^{p^{\prime}}\left(\mathbb{R}^{n}\right) \rightarrow$ $L^{p^{\prime}}\left(\mathbb{R}^{n}\right)$. But this is equivalent to $r>1$ by parts (i) and (ii) of the following theorem which is the corresponding version of Theorem 5.1 within the Lorentz spaces context. The proof can also be found in [20].

Theorem 5.2. Let $1<p, s<\infty$, and $1 \leq q<\infty$. Then the following are equivalent.

$$
s<p
$$

(ii) there is a constant $c$ such that

$$
\int_{\mathbb{R}^{n}} M_{s, q} f(y)^{p} d y \leq c \int_{\mathbb{R}^{n}} f(y)^{p} d y
$$

for all nonnegative, locally integrable functions $f$;

(iii) there is a constant $c$ such that

$$
\int_{\mathbb{R}^{n}} M_{s, q} f(y)^{p} w(y) d y \leq c \int_{\mathbb{R}^{n}} f(y)^{p} M w(y) d y
$$

for all nonnegative, locally integrable functions $f$, and $w$;

(iv) there is a constant $c$ such that

$$
\int_{\mathbb{R}^{n}} M f(y)^{p} \frac{w(y)}{\left[M_{s^{\prime}, q^{\prime}}\left(u^{1 / p}\right)(y)\right]^{p}} d y \leq c \int_{\mathbb{R}^{n}} f(y)^{p} \frac{M w(y)}{u(y)} d y,
$$

for all nonnegative, locally integrable functions $f, w$ and $u$. 
Proof of Lemma 2.10. If $q=p$, then $B \in B_{\min \{p, q\}}=B_{p}$, and the vectorvalued inequality (14) follows from equivalences (i) and (ii) of Theorem 5.1. For $q>p$ we have that $B \in B_{\min \{p, q\}}=B_{p}$. Observe that the vector-valued inequality for $q=\infty$ is obvious for every $B$. Now, since $B \in B_{p}$ the vectorvalued inequality still persists for $q=p$ by Theorem 5.1 again, and the case $q>p$ is obtained by interpolation. Now, if $1<q<p<\infty$ we can write

$$
\begin{aligned}
\left\|\left(\sum_{i=0}^{\infty}\left(M_{B} f_{i}\right)^{q}\right)^{1 / q}\right\|_{L^{p}}^{q} & =\left\|\sum_{i=0}^{\infty}\left(M_{B} f_{i}\right)^{q}\right\|_{L^{p / q}} \\
& =\sum_{i=0}^{\infty} \int_{\mathbb{R}^{n}}\left(M_{B} f_{i}(x)\right)^{q} g(x) d x,
\end{aligned}
$$

for some $0 \leq g \in L^{t}$ with unit norm, where $t=\left(\frac{p}{q}\right)^{\prime}$. Since this time we have that $B \in B_{q}$, Theorem 5.1 (iii) yields

$$
\left\|\left(\sum_{i=0}^{\infty}\left(M_{B} f_{i}\right)^{q}\right)^{1 / q}\right\|_{L^{p}}^{q} \leq C \sum_{i=0}^{\infty} \int_{\mathbb{R}^{n}} f_{i}(x)^{q} M g(x) d x .
$$

Therefore, by Hölder's inequality and the Hardy-Littlewood maximal theorem the last expression can be estimated by $\left\|\left(\sum_{i=0}^{\infty}\left(f_{i}\right)^{q}\right)^{1 / q}\right\|_{L^{p}}^{q}$, concluding the proof of the lemma.

Proof of Theorem 2.9. As in the proof of Theorem 2.3 we first claim that (13) is equivalent to

$$
\left\|\left(\sum_{i=0}^{\infty}\left|I_{1} f_{i}\right|^{q}\right)^{1 / q} v\right\|_{L^{p}} \leq c\left\|\left(\sum_{i=0}^{\infty}\left|f_{i}\right|^{q}\right)^{1 / q}\right\|_{L^{p}} .
$$

That this is sufficient follows from $f(x) \leq c I_{1}(|\nabla f|)(x), f \in C_{0}^{\infty}$. To show that it is also necessary we may use the same argument as in the proof of Theorem 2.3 but using this time the vector-valued inequality of $\mathrm{A}$. Córdoba and C. Fefferman (cf. [8])

$$
\left\|\left(\sum_{i=0}^{\infty}\left|K f_{i}\right|^{q}\right)^{1 / q} v\right\|_{L^{p}} \leq c\left\|\left(\sum_{i=0}^{\infty}\left|f_{i}\right|^{q}\right)^{1 / q}\right\|_{L^{p}} .
$$

Here $K$ is a classical Calderón-Zygmund singular integral operator as defined in [23].

To prove (35) we consider the Banach space $L^{p}\left(l^{q}\right)$. We recall that the space $L^{p}\left(l^{q}\right)$ consists of all sequences $\left(f_{i}\right)$ such that

$$
\left\|\left(f_{i}\right)\right\|_{L^{p}\left(l^{q}\right)}=\left\|\left(\sum_{i=0}^{\infty}\left|f_{i}\right|^{q}\right)^{1 / q}\right\|_{L^{p}}<\infty .
$$

Thus (35) is equivalent to saying that the operator

$$
T: L^{p}\left(l^{q}\right) \rightarrow L^{p}\left(l^{q}\right), \quad\left(f_{i}\right) \rightarrow\left(v I_{1} f_{i}\right)
$$


is bounded. Also this is the same as $T^{\prime}$ being bounded, where $T^{\prime}$ is the dual of $T$. However, since $I_{1}$ is selfadjoint the dual of $T$ is given by

$$
T^{\prime}: L^{p^{\prime}}\left(l^{q^{\prime}}\right) \rightarrow L^{p^{\prime}}\left(l^{q^{\prime}}\right), \quad\left(f_{i}\right) \rightarrow\left(I_{1}\left(v f_{i}\right)\right) .
$$

Hence, it is enough to show the following vector-valued inequality:

$$
\left\|\left(\sum_{i=0}^{\infty} I_{1}\left(v f_{i}\right)^{q^{\prime}}\right)^{1 / q^{\prime}}\right\|_{L^{p^{\prime}}} \leq c\left\|\left(\sum_{i=0}^{\infty}\left(f_{i}\right)^{q^{\prime}}\right)^{1 / q^{\prime}}\right\|_{L^{p^{\prime}}},
$$

for all functions $0 \leq f_{i} \in C_{0}^{\infty}$. Now, by the vector-valued version of the Muckenhoupt-Wheeden theorem (Theorem 2.2) this is also equivalent with

$$
\left\|\left(\sum_{i=0}^{\infty} M_{1}\left(v f_{i}\right)^{q^{\prime}}\right)^{1 / q^{\prime}}\right\|_{L^{p^{\prime}}} \leq c\left\|\left(\sum_{i=0}^{\infty}\left(f_{i}\right)_{q^{\prime}}\right)^{1 / q^{\prime}}\right\|_{L^{p^{\prime}}},
$$

for all functions $0 \leq f_{i} \in C_{0}^{\infty}$. Now, to estimate the left-hand side of (37) we observe as in the proof of Theorem 2.3 that (23) and (12) yield $M_{1}(v f)(x) \leq$ $k M_{\bar{B}} f(x)$, for each $x \in \mathbb{R}^{n}$. Hence, the left-hand side of (37) is bounded by

$$
k\left\|\left(\sum_{i=0}^{\infty} M_{\bar{B}}\left(f_{i}\right)^{q^{\prime}}\right)^{1 / q^{\prime}}\right\|_{L^{p^{\prime}}} .
$$

Finally, since we assume that $\bar{B} \in B_{\min \left\{p^{\prime}, q^{\prime}\right\}}$, an application of Lemma 2.10 concludes the proof of Theorem 2.9.

\section{REFERENCES}

1. D. Adams, Weighted nonlinear potential theory, Trans. Amer. Math. Soc. 297 (1986), 73-94.

2. R. Bagby and J. Parsons, Orlicz spaces and rearranged maximal functions, Math. Nachr. 132 (1987), 15-27.

3. C. Bennett and R. Sharpley, Interpolation of operators, Academic Press, New York, 1988.

4. J. Bruna and B. Korenblum, On Kolgomorov's theorem, the Hardy-Littlewood maximal function and the radial maximal function, J. Analyse Math. 50 (1988), 225-239.

5. A. P. Calderón, Inequalities for the maximal function relative to a metric, Studia Math. $\mathbf{5 7}$ (1976), 297-306.

6. S. Y. A. Chang, J. M. Wilson, and T. H. Wolff, Some weighted norm inequalities concerning the Schrödinger operators, Comment. Math. Helv. 60 (1985), 217-286.

7. S. Chanillo and R. L. Wheeden, $L^{p}$ estimates for fractional integrals and Sobolev inequalities with applications to Schrödinger operators, Comm. Partial Differential Equations 10 (1985), 1077-1116.

8. A. Córdoba and C. Fefferman, Weighted inequalities for singular integrals, Studia Math. $\mathbf{5 7}$ (1976), 97-101.

9. C. Fefferman, The uncertainty principle, Bull. Amer. Math. Soc. (N.S.) 9 (1983), 129-206.

10. C. Fefferman and E. M. Stein, Some maximal inequalities, Amer. J. Math. 93 (1971), 107115.

11. J. Garcia-Cuerva and J. L. Rubio de Francia, Weighted norm inequalities and related topics, North-Holland Math. Studies, no. 116, North-Holland, Amsterdam, 1985.

12. L. I. Hedberg and Th. H. Wolff, Thin sets in nonlinear potential theory, Ann. Inst. Fourier (Grenoble) 33 (1983), 161-187.

13. M. Giaquinta, Multiple integrals in the calculus of variations and nonlinear elliptic systems, Ann. of Math. Studies, no. 105, Princeton Univ. Press, Princeton, N.J., 1983. 
14. Y. Giga and T. Miyakawa, Navier-Stokes flow in $\mathbb{R}^{3}$ with measures as initial vorticity and Morrey spaces, Comm. Partial Differential Equations 14 (1989), 577-618.

15. B. Jawerth, C. Pérez and G. Welland, The positive cone in Triebel-Lizorkin spaces and the relation among potential and maximal operators, (M. Milman and T. Schonbek, eds.), Contemp. Math., vol. 107, Amer. Math. Soc., Providence, R.I., 1989.

16. R. Kerman and E. Sawyer, The trace inequality and eigenvalue estimates for Schrödinger operators, Ann. Inst. Fourier (Grenoble) 36 (1986), 207-228.

17. M. A. Krasnosel'skiĩ and J. B. Rutickii, Convex functions and Orlicz spaces, Noordhoff, Groningen, 1961.

18. A. Kufner, J. Oldriich and F. Svatopluk, Function spaces, Noordhoff, Leyden, 1977.

19. B. Muckenhoupt and R. L. Wheeden, Weighted norm inequalities for fractional integrals, Trans. Amer. Math. Soc. 191 (1974), 261-274.

20. C. Pérez, On sufficient conditions for the boundedness of the Hardy-Littlewood maximal operator between weighted $L^{p}$-spaces with different weights, preprint, 1990.

21. E. Sawyer and R. L. Wheeden, Weighted inequalities for fractional integrals on euclidean and homogeneous spaces, preprint, 1989.

22. M. Schechter, The spectrum of the Schrödinger operator, preprint, 1988.

23. E. M. Stein, Singular integrals of differentiability properties of functions, Princeton Univ. Press, Princeton, N.J., 1970.

24. E. M. Stein and G. L. Weiss, Introduction to Fourier analysis on Euclidean spaces, Princeton Univ. Press, Princeton, N.J., 1970.

25. J. M. Wilson, Weighted norm inequalities for the continuous square functions, Trans. Amer. Math. Soc. 341 (1989), 661-692.

Department of Mathematics, Brock University, St. Catharines, Ontario, Canada L2S $3 \mathrm{~A} 1$

Department of Mathematical Sciences, New Mexico State University, Las Cruces, New MeXico 88003

Current address: Departamento de Matematicas Universidad Autonoma de Madrid, 28049 Madrid, Spain 